\title{
Quantum information approach to the quantum phase transition in the Kitaev honeycomb model
}

\author{
Jian Cui. $*$ Jun-Peng Cao, and Heng Fan \\ Institute of Physics, Chinese Academy of Sciences, \\ Beijing National Laboratory for Condensed Matter Physics, Beijing 100190, China
}

(Dated: June 7, 2018)

\begin{abstract}
Kitaev honeycomb model with topological phase transition at zero temperature is studied using quantum information method. Based on the exact solution of the ground state, the mutual information between two nearest sites and between two bonds with longest distance are obtained. It is found that the mutual information shows some singularities at the critical point the system transits from gapless phase to gapped phase. The finite-size effects and scaling behavior are also studied. Our results indicate that the mutual information can serve as good indicator of the topological phase transition. This is because that the mutual information is believed to be able to catch some global correlation properties of the system. Meanwhile, this method has advantages that the phase transition can be determined easily and the order parameters, which are hard to be obtained for some topological phase transitions, are not necessarily known.
\end{abstract}

PACS numbers: 03.67.-a 05.70.Jk 05.30.Pr 75.10.Jm

\section{Introduction}

Recently, Kitaev honeycomb model has become a popular subject in both the fields of condensed matter physics and quantum information processing [1-13]. This model was first introduced by Kitaev to study the anyons, and the analytic exact solution to the ground state of this model has been obtained by several methods 1, 1417. It has rich phase transitions and has both a gapless phase with non-Abelian anyons excitation and three gapped phases with Abelian anyons excitations depending on the values of the parameters in the Hamiltonian. It is shown that the system possess a topological phase transition, which is not able to be characterized by symmetry breaking theory and the corresponding local order parameters but can be characterized by nonlocal string order parameters [1, 18]. One interesting point is that the system is a scarce exactly solvable model with dimensions higher than one, thus it provides a test bed for many numerical methods in two dimensional systems just as the Ising model does in one dimension. With these interesting properties, Kitaev honeycomb model has been studied intensively and is extended to other cases [12, 19].

On the other hand, Kitaev honeycomb model also has good practical advantages to be an active subject in that it has great potential applications in the quantum information and quantum computation. It was suggested to use the Kitaev honeycomb model to realize the faulttolerant topological quantum computation. The system is a good candidate to encode quantum information while those quantum states can be naturally protected from the inevitable decoherence by environment [20]. The Kitaev honeycomb model can be realized using the optical lattice 21, 22], and using the superconducting quantum

*Electronic address: cuijian@iphy.ac.cn circuits 23, 24. It is also studied by means of fidelity susceptibility [15] and the extended Kitaev model is studied by approaches of entanglement[13].

In this paper, we investigate the Kitaev honeycomb model from the quantum information perspective 25, 26]. We study the topological phase transition in this model by means of mutual information between the component lattices. It is generally believed that the mutual information measures the total information and describes the global correlation properties 27]. We find that both the derivative of mutual information between two nearest neighbor lattices and the mutual information between two bonds of the lattice can detect the topological phase transition in the Kitaev honeycomb model. This quantum information method has great advantages in that the singular behavior occurs exactly at the point when the gapless phase transits into a gapped phase. We also study the finite-size effects and the scaling behavior of the singularities of the mutual information.

This paper is organized as follows. In Section II we briefly introduce the Kitaev honeycomb model, then diagonalize the Hamiltonian and give the exact solution of the ground state based on the initial Kitaev's method. After that, we calculate the two sites and four sites correlation functions getting prepared for the two-site and two-bond reduced density matrix. In section III and section IV, we calculate the two-site mutual information and two-bond mutual information and the former one's derivative, respectively. Section $\mathrm{V}$ is the conclusions and remarks.

\section{Kitaev honeycomb model}

Kitaev honeycomb model is a two dimensional spin- $\frac{1}{2}$ lattice model with nearest neighbor interactions. It has two kinds of simple sublattice which are denoted by the dark dots and empty circles in figure 1. Each lattice in- 


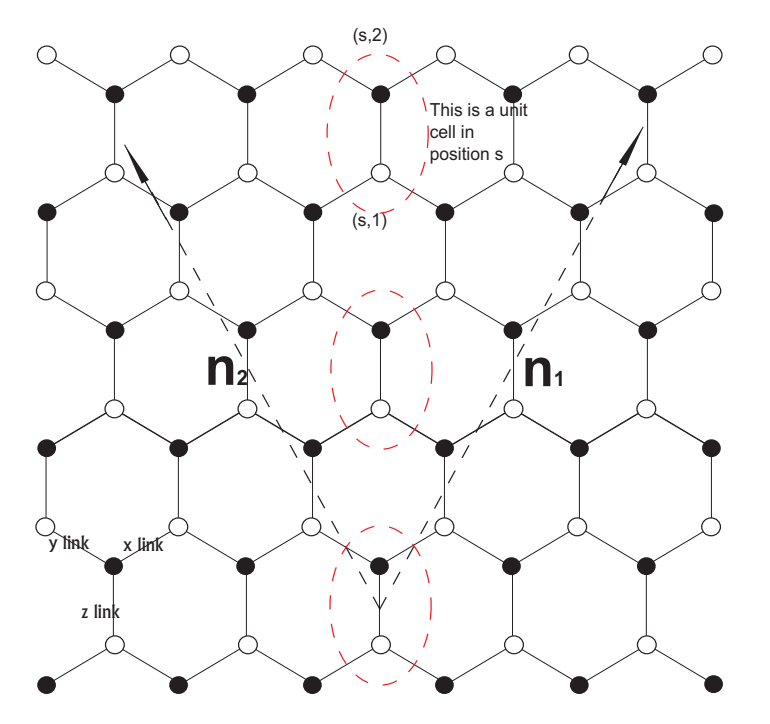

FIG. 1: (color online). The sketch map of Kitaev honeycomb model. The unit cell contains two sites of different kinds, which is highlighted by a elliptic circle. For simplicity, we choose the coordinate axes in $\mathbf{n}_{1}$ and $\mathbf{n}_{2}$ directions.

teracts with three nearest neighbors of the opposite kind through three distinct bonds labeled as $x$ link, $y$ link and $z$ link. For each bond the interaction has different coupling constant. The Hamiltonian is

$H=-J_{x} \sum_{x-\text { links }} \sigma_{j}^{x} \sigma_{k}^{x}-J_{y} \sum_{y-l i n k s} \sigma_{j}^{y} \sigma_{k}^{y}-J_{z} \sum_{z-l i n k s} \sigma_{j}^{z} \sigma_{k}^{z}$,

where the subindex $j, k$ denote the location of the site, and $\sigma_{k}^{\alpha}(\alpha=x, y, z)$ is the pauli matrix at site $k$. We take axis of the system in the $\mathbf{n}_{1}$ and $\mathbf{n}_{2}$ direction, and in each direction there are $L$ unit cells. Therefore the whole system has $2 L^{2}$ sites. Next, we used the original Kitaev's method to diagonalize this Hamiltonian and get its ground state.

\section{A. The ground state}

We first introduce the following Majorana transformation to transform the Pauli operators into the Majorana fermion operators.

$$
\sigma^{x}=i b^{x} c, \sigma^{y}=i b^{y} c, \sigma^{z}=i b^{z} c,
$$

where the Majorana operators satisfy $A^{\dagger}=A, A^{2}=1$, $A B+B A=0, b^{x} b^{y} b^{z} c=1$, for $A, B \in\left\{b^{x}, b^{y}, b^{z}, c\right\}$ and
$A \neq B$. Thus, the Hamiltonian becomes

$$
\begin{aligned}
H & =-\sum_{\alpha} J^{\alpha} \sum_{\alpha-l i n k s} b_{j}^{\alpha} b_{k}^{\alpha} c_{j} c_{k} \\
& =i \sum_{\alpha} J^{\alpha} \sum_{\alpha-l i n k s}\left(i b_{j}^{\alpha} b_{k}^{\alpha}\right) c_{j} c_{k} \\
& =i \sum_{\alpha} J^{\alpha} \sum_{\alpha-l i n k s} \hat{u}_{j k} c_{j} c_{k} \\
& =\frac{i}{2} \sum_{j, k} J_{\alpha_{j, k}} \hat{u}_{j, k} c_{j} c_{k} .
\end{aligned}
$$

In the last equation, the value of $\alpha$ is totally determined by the site index $j$ and $k$. The factor $\frac{1}{2}$ is due to the summation of the lattices has counted each lattice twice. It can be easily shown that $\hat{u}_{j, k}^{2}=1,\left[\hat{u}_{j, k}, H\right]=0$, and $\hat{u}_{j, k}$ commute with each other. As a result the eigenvalues of $\hat{u}_{j, k}$ here we present by $u_{j, k}$ are \pm 1 , and the whole Hilbert space can be decomposed into a series of eigenvalue spaces described by the eigenvalues of $\hat{u}_{j, k}$. According to [14, 28], the ground state is in the vortex free space so that we assume $u_{j, k}=1$ for all links, where $j$ is a kind of simple sublattice presented by the empty circles in this paper. Notice $u_{j, k}=-u_{k, j}$.

As the unit cell of this model contains one empty circle lattice and one dark dot lattice, we introduce a pair of index $(\mathbf{s}, \lambda)$ to take the place of the previous site index $j$, where the first index $\mathbf{s}$ stands for the location of the unit cell, and the second one describes the two different kinds of sublattice. In this paper, we let the empty circle's second index takes the value 1 , and the dark dot's takes the value 2. Please see figure 1. Then the Hamiltonian becomes

$$
H=\frac{i}{2} \sum_{\mathbf{s}, \lambda, \mathbf{t}, \mu} J_{\mathbf{s}, \lambda, \mathbf{t}, \mu} c_{\mathbf{s}, \lambda} c_{\mathbf{t}, \mu} .
$$

The two dimensional system we studied is on the surface of a torus with periodical boundary conditions. Because of the translational invariance of the system $J_{\mathbf{s}, \lambda, t, \mu}$ is actually determined by three index $\lambda, \mu$, and $\mathbf{t}-\mathbf{s}$. Then we introduce the Fourier transformation

$$
\begin{aligned}
J_{\mathbf{s}, \lambda ; \mathbf{t}, \mu} & =J_{0, \lambda ; \mathbf{t}-\mathbf{s}, \mu}=\frac{1}{L^{2}} \sum_{\mathbf{q}} e^{-i \mathbf{q} \cdot\left(\mathbf{r}_{t}-\mathbf{r}_{s}\right)} \widetilde{J_{\lambda, \mu}}(\mathbf{q}), \\
c_{\mathbf{s}, \lambda} & =\sqrt{\frac{2}{L^{2}}} \sum_{\mathbf{q}} e^{i \mathbf{q} \cdot \mathbf{r}_{s}} a_{\mathbf{q}, \lambda} .
\end{aligned}
$$

The inverse transformation is

$$
\begin{aligned}
\widetilde{J_{\lambda, \mu}}(\mathbf{q}) & =\sum_{\mathbf{t}} e^{i \mathbf{q} \cdot \mathbf{r}_{t}} J_{0, \lambda ; \mathbf{t}, \mu}, \\
a_{\mathbf{q}, \lambda} & =\sqrt{\frac{1}{2 L^{2}}} \sum_{\mathbf{s}} e^{-i \mathbf{q} \cdot \mathbf{r}_{s}} c_{\mathbf{s}, \lambda},
\end{aligned}
$$

where $a_{\mathbf{q}, \lambda}$ satisfies $a_{-\mathbf{q}, \lambda}=a_{\mathbf{q}, \lambda}^{\dagger}, a_{\mathbf{q}, \lambda}^{2}=0$, $\left[a_{\mathbf{q}, \lambda}, a_{\mathbf{q}, \mu}^{\dagger}\right]_{+} \equiv a_{\mathbf{q}, \lambda} a_{\mathbf{q}, \mu}^{\dagger}+a_{\mathbf{q}, \mu}^{\dagger} a_{\mathbf{q}, \lambda}=\delta_{\mathbf{p q}} \delta_{\lambda, \mu}$, and other 
anticommutators are all equal to zero. Then the Hamiltonian arrives at

$$
H=i \sum_{\mathbf{q}} \sum_{\lambda, \mu=1}^{2} \widetilde{J_{\lambda, \mu}}(\mathbf{q}) a_{-\mathbf{q}, \lambda} a_{\mathbf{q}, \mu} .
$$

After simple calculations we obtain that $\widetilde{J_{1,1}}(\mathbf{q})=$ $\sum_{t} e^{i \mathbf{q} \cdot \mathbf{r}_{t}} J_{01, \mathbf{t} 1}=0$, because $J_{01, \mathbf{t} 1}=0$. For the similar reason $\widetilde{J_{2,2}}(\mathbf{q})=0$. As each lattice interacts with its three nearest neighbors, there are only three values of $\mathbf{t}$ corresponding to the three neighbors that make $J_{01, \mathbf{t} 2}$ take nonzero values. Thus $\widetilde{J_{1,2}}(\mathbf{q})=J_{x} e^{i \mathbf{q} \cdot \mathbf{n}_{1}}+$ $J_{y} e^{i \mathbf{q} \cdot \mathbf{n}_{2}}+J_{z}$, and $\widetilde{J_{2,1}}(\mathbf{q})=-\widetilde{J_{1,2}}(\mathbf{q})$, where $\mathbf{n}_{1}$ and $\mathbf{n}_{2}$ are in certain directions which is shown in figure 1 . Let $f(\mathbf{q}) \equiv \widehat{J_{1,2}}(\mathbf{q})=\varepsilon(\mathbf{q})+i \Delta(\mathbf{q})$, and choose $\overrightarrow{q_{x}}$ to be in the direction of $\mathbf{n}_{1}$, and $\overrightarrow{q_{y}}$ to be in the direction of $\mathbf{n}_{2}$. Then we have

$$
\begin{aligned}
\varepsilon(\mathbf{q}) & =J_{x} \cos q_{x}+J_{y} \cos q_{y}+J_{z}, \\
\Delta(\mathbf{q}) & =J_{x} \sin q_{x}+J_{y} \operatorname{sinq} q_{y},
\end{aligned}
$$

where $q_{x}$ and $q_{y}$ take values $q_{x}, q_{y}=2 \pi n / L, n=$ $-(L-1) / 2, \cdots,(L-1) / 2$. We can see that $\varepsilon(-\mathbf{q})=\varepsilon(\mathbf{q})$, $\Delta(-\mathbf{q})=-\Delta(\mathbf{q})$, and $f(-\mathbf{q})=f^{*}(\mathbf{q})$. The Hamiltonian becomes

$$
H=\sum_{\mathbf{q}} i f(\mathbf{q}) a_{\mathbf{q}, 1}^{\dagger} a_{\mathbf{q}, 2}+(i f(\mathbf{q}))^{*} a_{\mathbf{q}, 2}^{\dagger} a_{\mathbf{q}, 1} .
$$

Next, we introduce the following Bogoliubov transformation:

$$
\begin{aligned}
& C_{\mathbf{q}, 1}=u_{\mathbf{q}} a_{\mathbf{q}, 1}+v_{\mathbf{q}} a_{\mathbf{q}, 2}, \\
& C_{\mathbf{q}, 1}^{\dagger}=u_{\mathbf{q}}^{*} a_{\mathbf{q}, 1}^{\dagger}+v_{\mathbf{q}}^{*} a_{\mathbf{q}, 2}^{\dagger}, \\
& C_{\mathbf{q}, 2}=v_{\mathbf{q}}^{*} a_{\mathbf{q}, 1}-u_{\mathbf{q}}^{*} a_{\mathbf{q}, 2}, \\
& C_{\mathbf{q}, 2}^{\dagger}=v_{\mathbf{q}} a_{\mathbf{q}, 1}^{\dagger}-u_{\mathbf{q}} a_{\mathbf{q}, 2}^{\dagger},
\end{aligned}
$$

with the new operators satisfying $\left[C_{\mathbf{q}, \lambda}, C_{\mathbf{p}, \mu}^{\dagger}\right]_{+}=$ $\delta_{\mathbf{p q}} \delta_{\lambda, \mu}, C_{\mathbf{q}, \lambda}^{2}=0$. Using the Bogoliubov transformation, the Hamiltonian is diagonalized as

$$
H=\sum_{\mathbf{q}}\left|f_{\mathbf{q}}\right|\left(C_{\mathbf{q}, 1}^{\dagger} C_{\mathbf{q}, 1}-C_{\mathbf{q}, 2}^{\dagger} C_{\mathbf{q}, 2}\right)
$$

with $u_{\mathbf{q}}=\frac{1}{\sqrt{2}}, v_{\mathbf{q}}=\frac{i}{\sqrt{2}} \frac{f_{\mathbf{q}}}{\left|f_{\mathbf{q}}\right|}, v_{-\mathbf{q}}=-v_{\mathbf{q}}^{*}, C_{-\mathbf{q}, 1}=$ $-2 u_{\mathbf{q}}^{*} v_{\mathbf{q}}^{*} C_{\mathbf{q}, 2}^{\dagger}$. Considering the fact $C_{\mathbf{q}, 1}^{\dagger} C_{\mathbf{q}, 1}=1-$ $C_{-\mathbf{q}, 2}^{\dagger} C_{-\mathbf{q}, 2}$, the Hamiltonian reads

$$
\begin{aligned}
H & =\sum_{\mathbf{q}}\left|f_{\mathbf{q}}\right|\left(1-C_{-\mathbf{q}, 2}^{\dagger} C_{-\mathbf{q}, 2}-C_{\mathbf{q}, 2}^{\dagger} C_{\mathbf{q}, 2}\right) \\
& =\sum_{\mathbf{q}}\left|f_{\mathbf{q}}\right|\left(1-2 C_{\mathbf{q}, 2}^{\dagger} C_{\mathbf{q}, 2}\right) .
\end{aligned}
$$

The normalized ground state is

$$
|G\rangle=\prod_{\mathbf{q}} C_{\mathbf{q}, 2}^{\dagger}|0\rangle,
$$

with $C_{\mathbf{q}, 2}|0\rangle=0$. The energy gap is $2 \min _{\mathbf{q}}\left\{\left|f_{\mathbf{q}}\right|\right\}$.

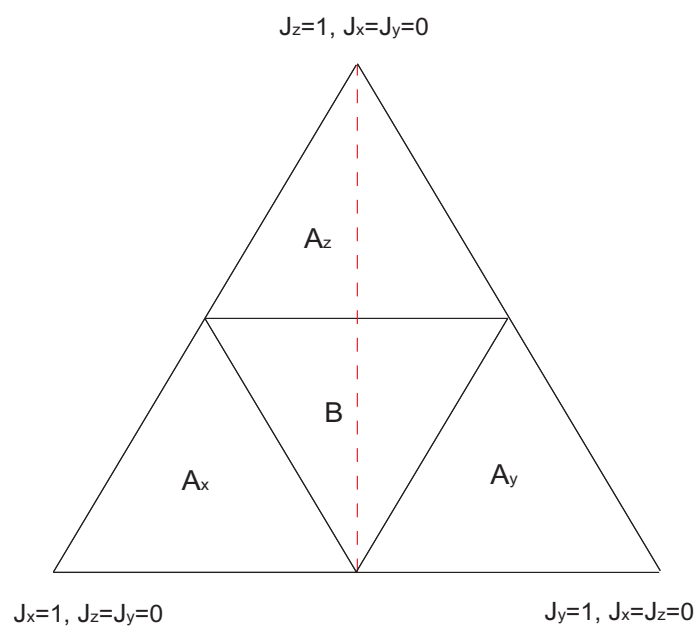

FIG. 2: (color online). The phase diagram of the Kitaev honeycomb model in the $J_{x}+J_{y}+J_{z}=1$ plane in the parameter space. In the three shadow areas labeled by $A_{x}, A_{y}$ and $A_{z}$, the system is gapped with Abelian anyon excitation, and in the blank area labeled by $B$ the system is gapless with non-Abelian excitation. In this paper, we focus on the red dash line $J_{x}=J_{y}=\left(1-J_{z}\right) / 2$, where the critical point of topological phase transition is $J_{z}=0.5$.

\section{B. Phase diagram}

This ground state has two distinct phases in the parameter space. In the region of $\left|J_{x}\right| \leq\left|J_{y}\right|+\left|J_{z}\right|$, $\left|J_{y}\right| \leq\left|J_{x}\right|+\left|J_{z}\right|$ and $\left|J_{z}\right| \leq\left|J_{y}\right|+\left|J_{x}\right|$ it is gapless with non-Abelian excitation and in other regions it is gapped with Abelian anyon excitations [14]. We focus on the $J_{x}+J_{y}+J_{z}=1$ plane. The phase diagram is shown in figure 2. In this paper, we investigate the behaviors of two-site mutual information and two-bond mutual information in the phase transition from the gapless phase to a gapped phase along the red dash line in the phase diagram of figure 2 .

\section{Correlation functions}

In this section we calculate the two-site and four-site correlation functions at the ground state of the systems which will be used to construct the reduced density matrix. Suppose the two nearest lattices to be studied are linked by $z$-bonds. The correlation function between two nearest lattices is

$$
\begin{aligned}
\left\langle\sigma_{\mathbf{r}, 1}^{z} \sigma_{\mathbf{r}, 2}^{z}\right\rangle & =\left\langle b_{\mathbf{r}, 1}^{z} b_{\mathbf{r}, 2}^{z} \frac{2}{L^{2}} \sum_{\mathbf{q}, \mathbf{q}^{\prime}} e^{i\left(\mathbf{q}+\mathbf{q}^{\prime}\right) \cdot \mathbf{r}} a_{\mathbf{q}, 1} a_{\mathbf{q}^{\prime}, 2}\right\rangle \\
& =-i \frac{2}{L^{2}} \sum_{\mathbf{q}, \mathbf{q}^{\prime}} e^{i\left(\mathbf{q}+\mathbf{q}^{\prime}\right) \cdot \mathbf{r}}\left\langle a_{\mathbf{q}, 1} a_{\mathbf{q}^{\prime}, 2}\right\rangle .
\end{aligned}
$$


By using the relation

$$
\begin{aligned}
\left\langle a_{\mathbf{q}, 1} a_{\mathbf{q}^{\prime}, 2}\right\rangle & =\left\langle\left(u_{\mathbf{q}}^{*} C_{\mathbf{q}, 1}+v_{\mathbf{q}} C_{\mathbf{q}, 2}\right)\left(v_{\mathbf{q}^{\prime}}^{*} C_{\mathbf{q}^{\prime}, 1}-u_{\mathbf{q}^{\prime}} C_{\mathbf{q}^{\prime}, 2}\right)\right\rangle \\
& =-u_{\mathbf{q}}^{*} u_{\mathbf{q}^{\prime}}\left\langle C_{\mathbf{q}, 1} C_{\mathbf{q}^{\prime}, 2}\right\rangle \\
& =\frac{i}{2} \delta_{\mathbf{q},-\mathbf{q}^{\prime}} \frac{f_{\mathbf{q}}}{\left|f_{\mathbf{q}}\right|}
\end{aligned}
$$

we obtain the correlation function

$$
\begin{aligned}
\left\langle\sigma_{\mathbf{r}, 1}^{z} \sigma_{\mathbf{r}, 2}^{z}\right\rangle & =\frac{1}{L^{2}} \sum_{\mathbf{q}} \frac{f_{\mathbf{q}}}{\left|f_{\mathbf{q}}\right|}=\frac{1}{2 L^{2}} \sum_{\mathbf{q}} \frac{f_{\mathbf{q}}+f_{-\mathbf{q}}}{\left|f_{\mathbf{q}}\right|} \\
& =\frac{1}{L^{2}} \sum_{\mathbf{q}} \frac{\varepsilon_{\mathbf{q}}}{E_{\mathbf{q}}}
\end{aligned}
$$

where $E_{\mathbf{q}}=\left|f_{\mathbf{q}}\right|=\sqrt{\varepsilon_{\mathbf{q}}^{2}+\Delta_{\mathbf{q}}^{2}}$.

The correlation function between two bonds as highlighted by elliptic circles in figure 1 is

$$
\begin{aligned}
\left\langle\sigma_{\mathbf{r}_{1}, 1}^{z} \sigma_{\mathbf{r}_{1}, 2}^{z} \sigma_{\mathbf{r}_{2}, 1}^{z} \sigma_{\mathbf{r}_{2}, 2}^{z}\right\rangle & =\left\langle b_{\mathbf{r}_{1}, 1}^{z} b_{\mathbf{r}_{1}, 2}^{z} b_{\mathbf{r}_{2}, 1}^{z} b_{\mathbf{r}_{2}, 2}^{z} C_{\mathbf{r}_{1}, 1} C_{\mathbf{r}_{1}, 2} C_{\mathbf{r}_{2}, 1} C_{\mathbf{r}_{2}, 2}\right\rangle \\
& =-\frac{4}{L^{4}} \sum_{\mathbf{q}_{1}, \mathbf{q}_{2}, \mathbf{q}_{3}, \mathbf{q}_{4}} e^{i\left(\mathbf{q}_{1}+\mathbf{q}_{2}\right) \cdot \mathbf{r}_{1}} e^{i\left(\mathbf{q}_{3}+\mathbf{q}_{4}\right) \cdot \mathbf{r}_{2}}\left\langle a_{\mathbf{r}_{1}, 1} a_{\mathbf{r}_{1}, 2} a_{\mathbf{r}_{2}, 1} a_{\mathbf{r}_{2}, 2}\right\rangle,
\end{aligned}
$$

where

$$
\begin{aligned}
\left\langle a_{\mathbf{r}_{1}, 1} a_{\mathbf{r}_{1}, 2} a_{\mathbf{r}_{2}, 1} a_{\mathbf{r}_{2}, 2}\right\rangle & =-\frac{1}{4} \frac{f_{\mathbf{q}_{1}}}{\left|f_{\mathbf{q}_{1}}\right|} \frac{f_{\mathbf{q}_{3}}}{\left|f_{\mathbf{q}_{3}}\right|}\left\langle C_{-\mathbf{q}_{1}, 2}^{\dagger}\left(C_{-\mathbf{q}_{2}, 2}+C_{\mathbf{q}_{2}, 2}\right)\left(C_{-\mathbf{q}_{3}, 2}^{\dagger}-C_{\mathbf{q}_{3}, 2}\right) C_{\mathbf{q}_{4}, 2}\right\rangle \\
& =\frac{1}{4} \frac{f_{\mathbf{q}_{1}}}{\left|f_{\mathbf{q}_{1}}\right|} \frac{f_{\mathbf{q}_{3}}}{\left|f_{\mathbf{q}_{3}}\right|}\left(\delta_{\mathbf{q}_{2},-\mathbf{q}_{3}} \delta_{\mathbf{q}_{1},-\mathbf{q}_{4}}-\delta_{\mathbf{q}_{1},-\mathbf{q}_{3}} \delta_{\mathbf{q}_{2},-\mathbf{q}_{4}}-\delta_{\mathbf{q}_{1},-\mathbf{q}_{2}} \delta_{\mathbf{q}_{3},-\mathbf{q}_{4}}\right) .
\end{aligned}
$$

Then, we arrive at

$$
\begin{aligned}
\left\langle\sigma_{\mathbf{r}_{1}, 1}^{z} \sigma_{\mathbf{r}_{1}, 2}^{z} \sigma_{\mathbf{r}_{2}, 1}^{z} \sigma_{\mathbf{r}_{2}, 2}^{z}\right\rangle & =-\frac{1}{L^{4}}\left(\sum_{\mathbf{q}_{1}, \mathbf{q}_{3}} \frac{f_{\mathbf{q}_{1}}}{\left|f_{\mathbf{q}_{1}}\right|} \frac{f_{\mathbf{q}_{3}}}{\left|f_{\mathbf{q}_{3}}\right|} e^{i\left(\mathbf{q}_{1}-\mathbf{q}_{3}\right) \cdot\left(\mathbf{r}_{1}-\mathbf{r}_{2}\right)}-\sum_{\mathbf{q}_{1}, \mathbf{q}_{2}} e^{i\left(\mathbf{q}_{1}+\mathbf{q}_{2}\right) \cdot\left(\mathbf{r}_{1}-\mathbf{r}_{2}\right)}-\sum_{\mathbf{q}_{1}, \mathbf{q}_{3}} \frac{f_{\mathbf{q}_{1}}}{\left|f_{\mathbf{q}_{1}}\right|} \frac{f_{\mathbf{q}_{3}}}{\left|f_{\mathbf{q}_{3}}\right|}\right) \\
& =-\frac{1}{L^{4}} \sum_{\mathbf{q}_{1}, \mathbf{q}_{3}} \frac{f_{\mathbf{q}_{1}} f_{\mathbf{q}_{3}}+f_{-\mathbf{q}_{1}} f_{-\mathbf{q}_{3}}}{\left|f_{\mathbf{q}_{1}}\right| \cdot\left|f_{\mathbf{q}_{3}}\right|}\left(\cos \left[\left(\mathbf{q}_{1}-\mathbf{q}_{3}\right) \cdot\left(\mathbf{r}_{1}-\mathbf{r}_{2}\right)\right]-1\right) \\
& =\frac{1}{L^{4}} \sum_{\mathbf{q}_{1}, \mathbf{q}_{3}} \frac{\Delta_{\mathbf{q}_{1}} \Delta_{\mathbf{q}_{3}}-\varepsilon_{\mathbf{q}_{1}} \varepsilon_{\mathbf{q}_{3}}}{E_{\mathbf{q}_{1}} E_{\mathbf{q}_{3}}}\left(\cos \left[\left(\mathbf{q}_{1}-\mathbf{q}_{3}\right) \cdot\left(\mathbf{r}_{1}-\mathbf{r}_{2}\right)\right]-1\right) .
\end{aligned}
$$

\section{Mutual information between two neighbor lattices}

The reduced density matrix of two site $i$ and $j$ is $\rho_{i, j}=\frac{1}{4} \sum_{\alpha, \beta=0}^{3}\left\langle\sigma_{i}^{\alpha} \sigma_{j}^{\beta}\right\rangle \sigma_{i}^{\alpha} \sigma_{j}^{\beta}$, where $\sigma^{1}=\sigma^{x}, \sigma^{2}=\sigma^{y}$, $\sigma^{3}=\sigma^{z}$ and $\sigma^{0}$ is identity. In the system (1), each site interacts with its three neighbors by different operators $\left(\sigma^{x}\right.$, $\sigma^{y}$ and $\sigma^{z}$ ), while each two linked sites together have only one kind of operator available, i.e., $\sigma^{\alpha} \sigma^{\alpha}$, with $\alpha$ corresponding to the type of their link. We find that only the correlation function along the link interacting direction is nonzero when we study the reduced matrix of two linked sites. That is to say if we consider the two lattices with $z$ link (please see figure 1), all the correlations are zero except $\left\langle\sigma^{z} \sigma^{z}\right\rangle$. Therefore, the reduced density matrix of this model has only diagonal elements, although the in- teractions of one site have three components. It indicates that the model (1) is much more like a classical system and similar to the Ising model. That may explain why this two dimensional model can be solved analytically.

In order to show our results more clearly, we use the numerical method to diagonalize the Hamiltonian exactly. With the periodical boundary conditions, we diagonalize an eight lattices system which is the smallest subsystem on the surface of a torus, please see figure 3 , and calculate all the 16 two-site correlation functions. Here we omit the subindex corresponding to the sites' position because the value is invariant due to the translational invariance of 


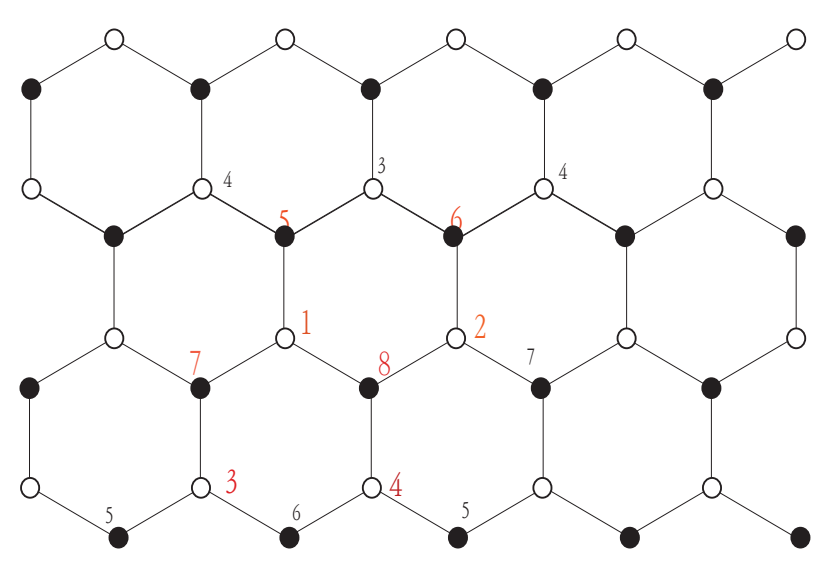

FIG. 3: (color online). The smallest translation invariant subsystem of Kitaev honeycomb model with periodical boundary conditions. The eight interaction sites in the subsystem are highlighted by red numbers in the graph. The sites labeled by small black numbers are the repetitions of the 8 sites because of the periodical boundary condition and torus topology.

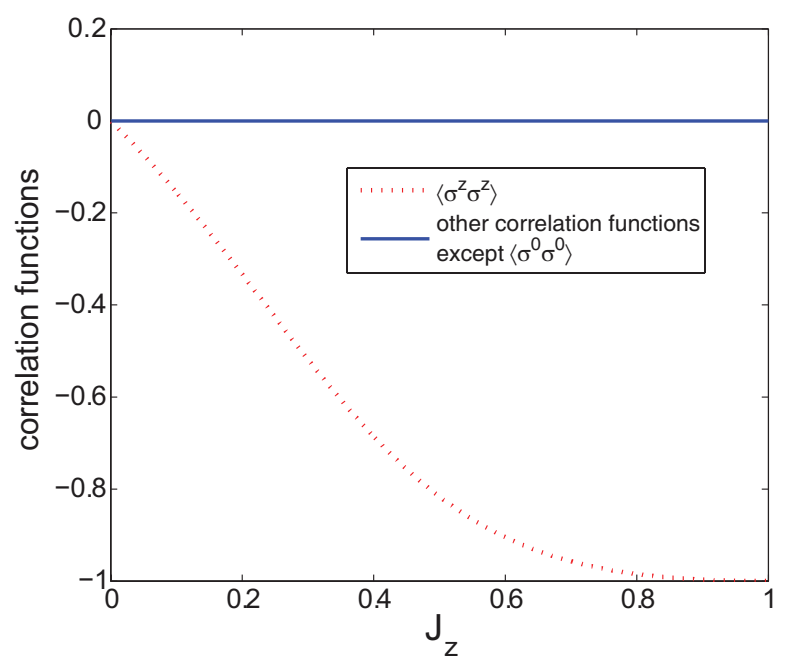

FIG. 4: (color online). The ground state correlation functions of two-site with $z$-link in the Kitaev honeycomb model. We see that $\left\langle\sigma_{\mathbf{r}, 1}^{0} \sigma_{\mathbf{r}, 2}^{0}\right\rangle=1,\left\langle\sigma_{\mathbf{r}, 1}^{z} \sigma_{\mathbf{r}, 2}^{z}\right\rangle \neq 0$ and others are zero. $J_{z}$ is in unit of $J_{x}+J_{y}+J_{z}$. this system. The explicit form of the Hamiltonian reads

$$
\begin{aligned}
H_{8} & =J_{x}\left(\sigma_{5}^{x} \sigma_{3}^{x}+\sigma_{6}^{x} \sigma_{4}^{x}+\sigma_{7}^{x} \sigma_{1}^{x}+\sigma_{8}^{x} \sigma_{2}^{x}\right) \\
& +J_{y}\left(\sigma_{3}^{y} \sigma_{6}^{y}+\sigma_{5}^{y} \sigma_{4}^{y}+\sigma_{8}^{y} \sigma_{1}^{y}+\sigma_{7}^{y} \sigma_{2}^{y}\right) \\
& +J_{z}\left(\sigma_{3}^{x} \sigma_{7}^{x}+\sigma_{4}^{x} \sigma_{8}^{x}+\sigma_{5}^{x} \sigma_{1}^{x}+\sigma_{6}^{x} \sigma_{2}^{x}\right)
\end{aligned}
$$

By using the periodical boundary conditions, the systemsize of the system (16) can be extend to infinity. The main properties of the system are kept since all possible interactions are considered. The result is shown in figure 4. We see that the correlation functions along the $z$ direction is non-zero.

The explicit form of reduced density matrix of two sites with nearest neighbor is

$$
\rho_{\mathbf{r}, 1 ; \mathbf{r}, 2}=\frac{1}{4}\left(\begin{array}{cccc}
1+\left\langle\sigma_{\mathbf{r}_{1}, 1}^{z} \sigma_{\mathbf{r}_{1}, 2}^{z}\right\rangle & 0 & 0 & 0 \\
0 & 1-\left\langle\sigma_{\mathbf{r}_{1}, 1}^{z} \sigma_{\mathbf{r}_{1}, 2}^{z}\right\rangle & 0 & 0 \\
0 & 0 & 1-\left\langle\sigma_{\mathbf{r}_{1}, 1}^{z} \sigma_{\mathbf{r}_{1}, 2}^{z}\right\rangle & 0 \\
0 & 0 & 0 & 1+\left\langle\sigma_{\mathbf{r}_{1}, 1}^{z} \sigma_{\mathbf{r}_{1}, 2}^{z}\right\rangle
\end{array}\right) .
$$

The eigenvalues of the reduced density matrix are $\lambda_{1}=\quad \lambda_{2}=\left(1-\left\langle\sigma^{z} \sigma^{z}\right\rangle\right) / 4$ and $\lambda_{3}=\lambda_{4}=\left(1+\left\langle\sigma^{z} \sigma^{z}\right\rangle\right) / 4$. Ev- 


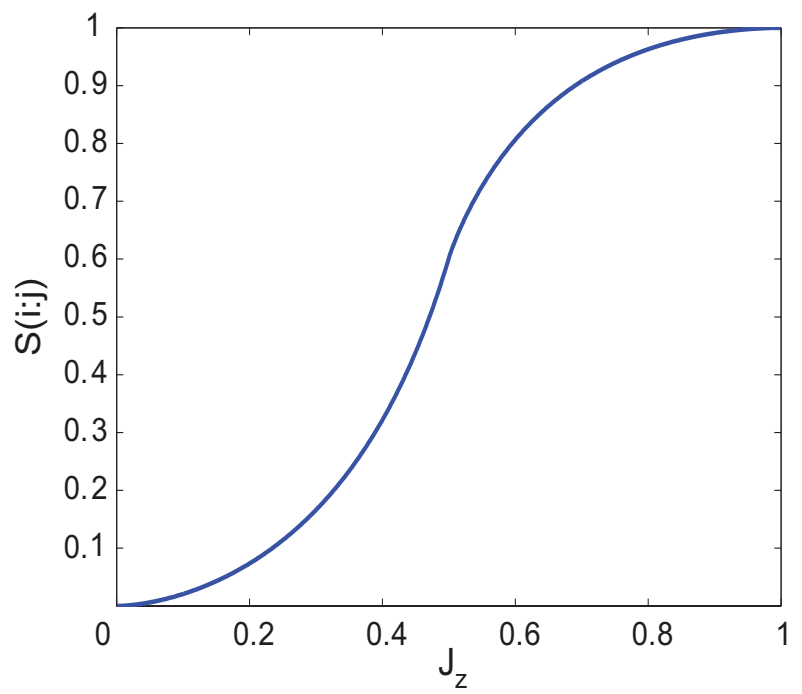

FIG. 5: (color online). Mutual information between two connected sites. The mutual information increases monotonically with the increasing of $J_{z}$. However, there exist a certain value $J_{z}^{m}$. If $J<J_{z}^{m}$, the mutual information is a concave function while if $J>J_{z}^{m}$, the mutual information is a convex function. Thus, the first order derivative of the mutual information with respect to $J_{z}$ has a peak at the $J_{z}^{m} . J_{z}$ is in unit of $J_{x}+J_{y}+J_{z}$.

ery eigenvalue corresponds the two-fold degenerate eigenstates. From the two sites reduced density matrix we can derive the reduced density matrix for one site as $I / 2$, where $I$ is identity matrix. The mutual information of the two sites $i$ and $j$ is

$$
S(i: j)=2-2 H\left(\frac{1-\left\langle\sigma^{z} \sigma^{z}\right\rangle}{4}\right)-2 H\left(\frac{1+\left\langle\sigma^{z} \sigma^{z}\right\rangle}{4}\right)
$$

where $H(x)=-x \log _{2}(x)$.

The the two-site mutual information along the line $J_{x}=J_{y}=\left(1-J_{z}\right) / 2$ is shown in figure 5 . We see that the mutual information increases monotonically with the increasing of $J_{z}$. However, there exist a certain value $J_{z}^{m}$. If $J<J_{z}^{m}$, the mutual information is a concave function while if $J>J_{z}^{m}$, the mutual information is a convex function. Thus, the first order derivative of the mutual information with respect to $J_{z}$ has a peak at the $J_{z}^{m}$. The derivative of the mutual information is shown in figure 6 . From figure 6 , we see that the first order derivative of the mutual information arrives at the maximum value at the point $J_{z}^{m}=0.5$, which exactly corresponds the critical point $J_{z}=0.5$. We also find that the value of $J_{z}^{m}$ is fixed when the system-size changes. The maximum value is a constant when the system size tends to infinity, as shown in figure 7 . This is different from the Ising model where the second order derivative of entanglement entropy diverges at the critical point in the the thermodynamic limit [31], although their density matrixes are same in structure.

From the quantum information perspective, the entanglement measured by concurrence between two sites is

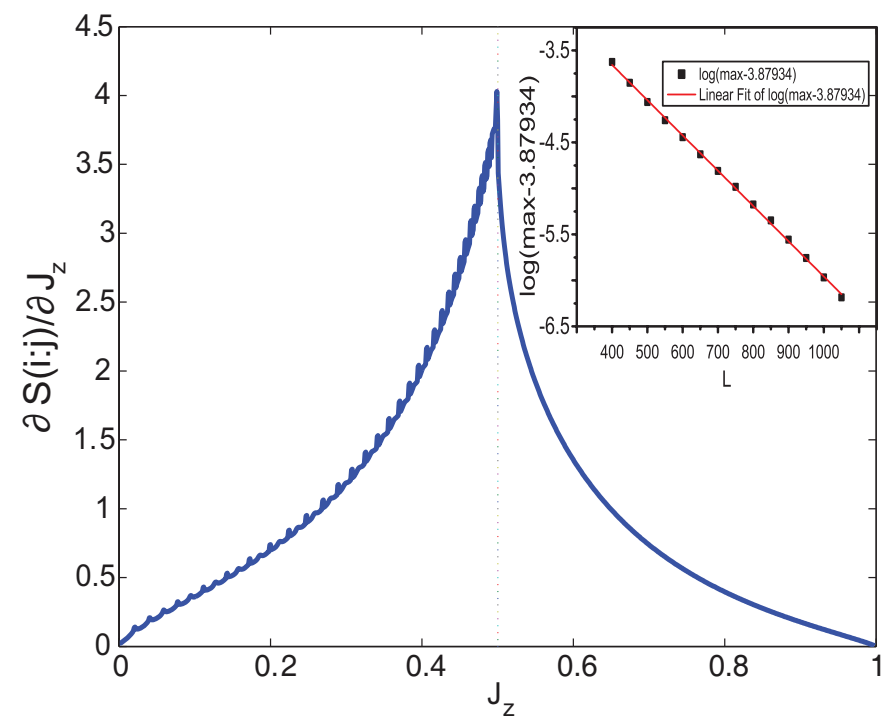

FIG. 6: (color online). The first order derivative of mutual information between two connected sites for the system-size $L=100$. The derivative of the mutual information has a maximum exactly at the critical point $J_{z}=0.5$. The small peaks in the gapless region is due to that while calculating the $\left\langle\sigma_{\mathbf{r}, 1}^{z} \sigma_{\mathbf{r}, 2}^{z}\right\rangle$ certain $\varepsilon_{\mathbf{q}}$ and $E_{\mathbf{q}}$ are both infinitesimal and some systemical error are included. The peaks get smaller when $L$ increases. The subgraph shows that when the system-size tends to infinity, the maximum of the derivative of the mutual information tends to a constant as $\log _{2}\left(\left(\frac{\partial S(i: j)}{\partial J_{z}}\right)_{\max }-3.87934\right)=-0.00384 L-2.12174 . \quad J_{z}$ is in unit of $J_{x}+J_{y}+J_{z}$.

zero since the density has only diagonal elements, while the entanglement between one site and all the rest sites is maximum entanglement, i.e., $c=\sqrt{\frac{d}{d-1}\left(1-\operatorname{Tr} \rho_{i}^{2}\right)}=1$, where $c$ denotes the concurrence, $\rho_{i}$ is the reduced density matrix of the particle in site $i$ and $d$ is the dimension of $\rho_{i}[29,30]$.

\section{Mutual information between two bonds with longest distance}

In this section, we investigate the mutual information between two $z$ linked bonds. Firstly, we need to calculate the density matrix. For arbitrary two $z$ linked bonds at 


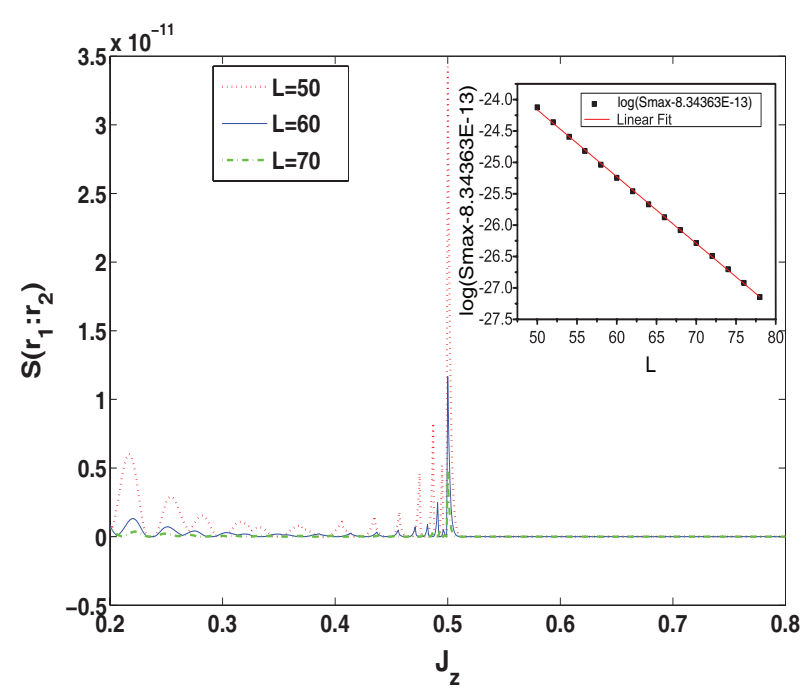

FIG. 7: (color online). The mutual information between two $z$ linked bonds with longest distance located on the torus surface for a given system-size. The small peaks in the gapless phase is caused by the $0 / 0$ error as before. The significant peak arises exactly at the critical point. The subgraph shows that when the system-size tends to infinity, the peak value of the mutual information trends to a constant as $\log _{2}\left(S\left(\mathbf{r}_{1}: \mathbf{r}_{2}\right)_{\max }-8.34363 \times 10^{-13}\right)=-0.10637 L-18.8454$. Here $J_{z}$ is in unit of $J_{x}+J_{y}+J_{z}$.

$\mathbf{r}_{1}$ and $\mathbf{r}_{2}$ the density matrix is

$$
\begin{gathered}
\rho_{\mathbf{r}_{1}, \mathbf{r}_{2}}=\frac{1}{16} \sum_{\alpha, \beta=0,3} \sigma_{\mathbf{r}_{1}, 1}^{\alpha} \sigma_{\mathbf{r}_{1}, 2}^{\alpha} \sigma_{\mathbf{r}_{2}, 1}^{\beta} \sigma_{\mathbf{r}_{2}, 2}^{\beta} \\
\times\left\langle\sigma_{\mathbf{r}_{1}, 1}^{\alpha} \sigma_{\mathbf{r}_{1}, 2}^{\alpha} \sigma_{\mathbf{r}_{2}, 1}^{\beta} \sigma_{\mathbf{r}_{2}, 2}^{\beta}\right\rangle .
\end{gathered}
$$

The eigenvalues of this density matrix are $(1-$ $\left.4\left\langle\sigma^{z} \sigma^{z} \sigma^{z} \sigma^{z}\right\rangle\right) / 16,\left(1-2\left\langle\sigma^{z} \sigma^{z}\right\rangle+\left\langle\sigma^{z} \sigma^{z} \sigma^{z} \sigma^{z}\right\rangle\right) / 16,(1+$ $\left.2\left\langle\sigma^{z} \sigma^{z}\right\rangle+\left\langle\sigma^{z} \sigma^{z} \sigma^{z} \sigma^{z}\right\rangle\right) / 16$. These eigenvalues correspond the 8 fold, 4 fold and 4 fold degenerate eigenstates, respectively. Then we obtain the mutual information between two $z$ linked bonds in $\mathbf{r}_{1}$ and $\mathbf{r}_{2}$ as

$$
\begin{aligned}
S\left(\mathbf{r}_{1}: \mathbf{r}_{2}\right) & =4 H\left(\frac{1+\left\langle\sigma^{z} \sigma^{z}\right\rangle}{4}\right)+4 H\left(\frac{1-\left\langle\sigma^{z} \sigma^{z}\right\rangle}{4}\right) \\
& -8 H\left(\frac{1-\left\langle\sigma^{z} \sigma^{z} \sigma^{z} \sigma^{z}\right\rangle}{16}\right) \\
& -4 H\left(\frac{1-2\left\langle\sigma^{z} \sigma^{z}\right\rangle+\left\langle\sigma^{z} \sigma^{z} \sigma^{z} \sigma^{z}\right\rangle}{16}\right) \\
& -4 H\left(\frac{1+2\left\langle\sigma^{z} \sigma^{z}\right\rangle+\left\langle\sigma^{z} \sigma^{z} \sigma^{z} \sigma^{z}\right\rangle}{16}\right) .
\end{aligned}
$$

In order to reveal the long range correlation in the system, we study the bonds in the direction that contains the largest distance in the torus, which is marked by a line of elliptic circles in figure 1. Without losing generality, we choose the positions of the bonds as $\mathbf{r}_{1}=(0,0)$ and $\mathbf{r}_{2}=(0.5 L, 0.5 L)$ in the $\left(\mathbf{n}_{1}, \mathbf{n}_{2}\right)$ coordinate system so that the two bonds are longest separated. The mutual information between two $z$ linked bonds in this direction is shown in figure 7 . We find that the mutual information has a maximum at the critical point. This property is valid for different system-sizes. The peak values tend to a constant when the system-size $L$ trends to infinity.

\section{Conclusions}

In this paper, based on the exact ground state of Kitaev honeycomb model we have obtained both the reduced density matrices of two nearest neighbor sites and between two $z$ linked bonds with longest distance. We show that these density matrices have only diagonal elements, so that there is no entanglement between two local sites or two local bonds, but the nonlocal entanglement between one site and the rest of the whole system is maximum. From quantum information theory, the ground state which is a multipartite state seems more like Greenberger-Horne-Zeilinger (GHZ) states other than W-type states. We have calculated the mutual information between two nearest neighbor sites and the mutual information between two $z$ linked bonds with the longest distance in the torus topology. The first order derivative of the former mutual information and the latter mutual information itself have peak at the point where the ground state transits from the gapless phase into a gapped phase. The above singular behavior serves as an exact and easily obtaining detector of the topological phase transition in the Kitaev honeycomb model. The so called localizable entanglement is related with the string order parameters and the hidden topological long range order in one dimension spin chains [32, 33]. More over, the topological phase and topological phase transition have their roots in the hidden topological long range order and the string order parameters. Therefore, the investigation of the relation among the localizable entanglement, topological phase transition and other related quantities in the exactly solvable two dimensional Kitaev model may greatly enhance our understanding of the topological phase. The research in this direction should be further explored to extensively study the topological phase and topological order by means of quantum information.

\section{Acknowledgement}

This work is supported by grants of National Natural Science Foundation of China and "973" program of Ministry of Science and Technology (MOST), China. 
[1] X. Y. Feng, G.M. Zhang, T. Xiang, Phys. Rev. Lett. 98, 087204 (2007).

[2] D. H. Lee, G. M. Zhang, T. Xiang, Phys. Rev. Lett. 99, 196805 (2007).

[3] G. Baskaran, S. Mandal, R. Shankar, Phys. Rev. Lett. 98, 247201 (2007).

[4] S. Mondal, D. Sen, K. Sengupta, Phys. Rev. B. 78, 045101 (2008).

[5] K.P. Schmidt, S. Dusuel, J. Vidal, Phys. Rev. Lett. 100, 057208 (2008)

[6] S. Dusuel, K.P. Schmidt, J. Vidal, Phys. Rev. Lett. 100, 177204 (2008)

[7] J. Vidal, K.P. Schmidt, S. Dusuel, Phys. Rev. B 78, 245121 (2008)

[8] G. Kells, J. K. Slingerland, J. Vala, Phys. Rev. B 80, 125415 (2009).

[9] J. H. Zhao, H. Q. Zhou, Phys. Rev. B 80, 014403 (2009).

[10] D. F. Abasto, P. Zanardi, Phys. Rev. A 79, 012321 (2009).

[11] G. Kells, A. T. Bolukbasi, V. Lahtinen, J. K. Slingerland, J. K. Pachos, J. Vala, Phys. Rev. Lett. 101, 240404 (2008).

[12] S. Mandal, N. Surendran, Phys. Rev. B 79, 024426 (2009).

[13] X. F. Shi, Y. Yu, J. Q. You, F. Nori, Phys. Rev. B 79, 134431 (2009).

[14] A. Kitaev, Ann.phys. 321, 2 (2006).

[15] S. Yang, S. J. Gu, C. P. Sun, H. Q. Lin, Phys. Rev. A 78, 012304 (2008).

[16] H. D. Chen and Z. Nussinov, J. Phys. A 41, 075001 (2008)

[17] Z. Nussinov and G. Ortiz, Phys. Rev. B 79, 214440 (2009)
[18] X. G. Wen, Quantum Field Theory of Many-Body Systems(Oxford University, New York,2004).

[19] H. Yao, S. A. Kivelson, Phys. Rev. Lett. 99, 247203 (2007).

[20] A. Kitaev, Ann. Phys. 303, 2 (2003).

[21] A. Micheli, G. K. Brennen, P. Zoller, Nat. Phys. 2, 341 (2006).

[22] L.M. Duan, E. Demler, M. D. Lukin, Phys. Rev. Lett. 91, 090402 (2003).

[23] Z. Y. Xue, S. L. Zhu, J. Q. You, Z. D. Wang, Phys. Rev. A 79, 040303(R) (2009).

[24] J. Q. You, X. F. Shi, F. Nori, Phys. Rev. B 81,014505 (2010).

[25] L. Amico, R. Fazio, A. Osterloh, V. Vedral, Rev. Mod. Phys. 80, 517 (2008).

[26] M.A. Nielsen, I.L. Chuang, Quantum computation and quantum information. (Cambridge: Cambridge University Press, 2000).

[27] B. Groisman , S. Popescu, A. Winter, Phys. Rev. A 72, 032317 (2005).

[28] E. H. Lieb, Phys. Rev. Lett. 73, 2158 (1994).

[29] S. Albererio, S. M. Fei , J. Opt. B. 3, 223 (2001); H. Fan, K. Matsumoto, H. Imai, J. Phys. A: Math. Gen. 36, 4151 (2003).

[30] W. Dür, G. Vidal, J. I. Cirac, Phys. Rev. A. 62, 062314 (2000).

[31] J. Cao, X. Cui, Z. Qi, W. Lu, Q. Niu and Y. Wang, Phys. Rev. B 75, 172401 (2007).

[32] M. Popp, F. Verstraete, M. A. Martin-Delgado, J. I. Cirac, Phys. Rev. A 71, 042306 (2005).

[33] H. Fan, Z. D. Wang, V. Vedral, arXiv:0903.3791v1(2009). 